\title{
ON THE BOUNDARY BEHAVIOR OF CONFORMAL MAPS*)
}

\author{
S.E. WARSCHAWSKI \\ To Professor Kiyoshi Noshiro on his 60th birthday
}

INTRODUGTION. Suppose $\Omega$ is a simply connected domain which is mapped conformally onto a disk. A much studied problem is the behavior of the mapping function at an accessible boundary point $\mathrm{P}$ of $\Omega$, in particular the question, under what conditions the map is "conformal" at such a point (a) in the sense that angles are preserved as $\mathrm{P}$ is approached from $\Omega$ ("semiconformality" at P) and (b) the dilatation at $\mathrm{P}$ is finite and positive. In his fundamental paper [8] in 1936, A. Ostrowski established a necessary and sufficient condition (depending on the geometry of the domain only) for the validity of the first property which subsumes all previous results and establishes a definitive solution of this problem. There has been extensive work on problem (b) (see [7] and [5, Chapters IV and VI]); in particular, a number of criteria have been obtained for the existence of the "angular derivative" of the mapping function and of the derivative for "unrestricted approach" (see [10]). In establishing a connection between angular and unrestricted derivatives a second theorem in [8], Ostrowski's "Faltensatz" plays an important role. The "Faltensatz" is a generalization of an earlier result [10] of the author for Jordan domains, which he used to deduce the existence of the unrestricted derivative from that of the angular derivative.

Ostrowski's proof of his two theorems are based on the systematic use of the harmonic measure and requires a rather extensive study of its properties. Later on another proof utilizing an entirely different method (Carathéodory's convergence theorem on the mapping functions of domains tending to a kernel) was given by J. Lelong Ferrand [4]; [5, Chapter IV]. In the present paper we use a method of $\mathrm{J}$. Wolff [11], abstracted in our Lemma 1, to give new

Received July 29, 1966.

Received August 15, 1966.

*) The preparation of this paper was sponsored (in part) by the Office of Naval Research under contract Nonr-2216(28) (NR-043-332) with the University of California. 
and particulary short proofs of these two theorems as well as several related results, Theorems la, 1b, 2 and 3. Subsequently we use our Theorem 2 to obtain an asymptotic expression for the mapping function at a boundary point (Theorem 4) and a new and quite general criterion for the existence of the angular derivative (Theorems 5 and 6). It is convenient for our purposes to relate all considerations to mapping of infinite strips.

1. A Lemma of J. Wolff. Let $S$ be a simply connected domain in the $w$ plane, $w=u+i v$, whose boundary contains the point $w=\infty$. Suppose that the infinite segment $L:\left\{u \geqq u_{0}, v=0\right\}$ of the real axis is contained in $S$. Let $w_{\infty}$ denote the boundary point of $S$ at $w=\infty$ which is accessible along $L$ as $u \rightarrow+\infty$. For $u \geqq u_{0}$ let $\theta_{u}$ denote the largest open segment on the line $\operatorname{Re}(w)=u$ which intersects the real axis and is contained in $S$, and $\theta(u)(\leqq \infty)$ its length. We assume that for $u>u_{0}, \theta_{u}$ separates the point $w_{0}=u_{0}$ from $w_{\infty}$, i.e. the crosscut $\theta_{u}$ divides $S$ into two subdomains such that $u_{0}$ is in one and $w_{\infty}$ is on the boundary of (strictly) the other of these two domains.

Following Ostrowski [8, p. 450] we denote the subdomain $K:\left\{w \mid w \in \theta_{u}\right.$, $\left.u \geqq u_{0}\right\}$ as the kernel of $S$. $K$ depends on the choice of $u_{0}$, but this is not essential for our purposes.

Suppose the function $z=Z(w)=X(w)+i Y(w)$ maps $S$ conformally onto the strip $\Sigma:\left\{z=x+i y|| y \mid<\frac{\pi}{2},-\infty<x<+\infty\right\}$ such that $\lim _{u \rightarrow \infty} X(u)=+\infty$. By standard results on boundary correspondence, for all sufficiently large $u, \theta_{u}$ is mapped onto an $\operatorname{arc} \sigma_{u} \subset \Sigma$ which connects a (finite) point on $y=\frac{\pi}{2}$ to a (finite) point on $y=-\frac{\pi}{2}$. We assume that $u_{0}$ is taken so large that this is satisfied for $u \geqq u_{0}$. The cross-cut $\theta_{u_{0}}$ divides $S$ into two subdomains; let $S_{0}$ denote the one which contains the part of the real axis for which $u>u_{0}$.

Let $\alpha$ be a (finite) endpoint of a $\theta_{u}$ for $u>u_{0}$. We describe a circle $C_{\rho}$ of radius $\rho$ about $\alpha$ where $\rho<\operatorname{Min}\left(u-u_{0},|\alpha-u|\right)$ so that $C_{\rho}$ does not intersect $\theta_{u_{0}}$ and the $u$-axis. $C_{\rho}$ crosses $\theta_{u}$ at a point $\alpha^{\prime}$. Let $k_{\rho}$ denote the largest (open) arc of $C_{\rho}$ which contains $\alpha^{\prime}$ and is contained in $S$; it is also contained in $S_{0} ; k_{\rho}$ divides $S_{0}$ into two domains and the one containing the segment $\alpha \alpha^{\prime}$ of $\theta_{u}$ will be denoted by $D_{\rho}$.

Lemma $1^{1)}$. For every $\delta, 0<\delta<\operatorname{Min}\left(e^{-32}, u-u_{0},|\alpha-u|\right)$ there exists a $\rho$,

1) This lemma is implicit in J. Wolff's proof of his theorem on the preservation of angles 
$\delta^{2}<\rho<\delta$, such that the image of $D_{\rho}$ under the mapping $w \rightarrow Z(w)$ is a domain $\Delta_{\rho}$ bounded by the image of $k_{\rho}$ and a finite segment of the line $y=\frac{\pi}{2}$ or $y=-\frac{\pi}{2}$. The diameter of $\Delta_{\rho}$ does not exceed $M\left(\log \frac{1}{\delta}\right)^{-\frac{1}{2}}$ where $M \leqq 6 \pi \sqrt{2}$.

An immediate consequence is the

Corollary. If $w_{1}, w_{2} \in D_{\rho}$, then

$$
\left|X\left(w_{1}\right)-X\left(w_{2}\right)\right| \leqq \frac{M}{\sqrt{\log \frac{1}{\delta}}}, \quad\left|Y\left(w_{1}\right)-Y\left(w_{2}\right)\right| \leqq \frac{M}{\sqrt{\log \frac{1}{\delta}}} .
$$

In particular, if we let $w_{1} \rightarrow \alpha$ along $\theta_{u}$ the second inequality implies that for $w \in D_{\rho}$

$$
\left|\frac{\pi}{2}-\right| Y(w)|| \leqq \frac{M}{\sqrt{\log \frac{1}{\delta}}} .
$$

For the sake of completeness we present the proof of this lemma. Let $C_{\rho}$ be a circle about $\alpha$ of radius $\rho$ and $k_{\rho}$ the arc of $C_{\rho}$ as described above. The image of $k_{\rho}$ under the mapping $w \rightarrow Z(w)$ is an arc $\gamma_{\rho}$ whose endpoints are two different points of the boundary of $\Sigma$. Since $k_{\rho}$ does not intersect $\theta_{u_{0}}$ and $L, \gamma_{\rho}$ does not meet $\sigma_{u_{0}}$ and the image $\Lambda$ of $L$. Consequently both endpoints of $\gamma_{\rho}$ are on $y=\frac{\pi}{2}$ or on $y=-\frac{\pi}{2}$. The image $\Delta_{\rho}$ of $D_{\rho}$ is the interior of the closed Jordan curve formed by $\gamma_{\rho}$ and the segment of $y=-\frac{\pi}{2}$ or $y=-\frac{\pi}{2}$-between its endpoints. The image $\beta$ of the (accessible) boundary point $\alpha$ is located on this segment.

Let $\beta=b+i \frac{\pi}{2}$. We consider for $w \in S$ the function

$$
\omega=\Omega(w)=\frac{e^{Z(w)}-e^{b}}{e^{Z(w)}+e^{b}},
$$

which is holomorphic and univalent, and $|\Omega(w)|<1$ in $S$. Furthermore, as $w \rightarrow \alpha$ along $\theta_{u}, \Omega(w) \rightarrow i$. The image $\kappa_{\rho}$ of $k_{\rho}$ under the mapping $w \rightarrow \omega$ is an arc in $|\omega|<1$ (except for its endpoints). By an earlier lemma of Wolff $[11$, p. 46] for every $\delta$ subject to the restrictions stated above there exists a $\rho, \delta^{2}<\rho<\delta$, such that for this value of $\rho$ the length of $\kappa_{\rho}$,

at a boundary point [11, pp. 47-49]. It can be given a more general form, for example, $\alpha$ may be an arbitrary accessible (finite) boundary point and $S$ may be a more general region. We present it in a form tailored to our immediate application. 


$$
\lambda_{\rho} \leqq \frac{\pi \sqrt{2}}{\sqrt{\log -\frac{1}{\delta}}} .
$$

In order to obtain an estimate for the length of $\gamma_{\rho}$ we note that $\kappa_{\rho}$ is the image of $\gamma_{\rho}$ under the mapping

$$
\omega=\frac{e^{z}-e^{b}}{e^{z}+e^{b}}
$$

This function is holomorphic and univalent in $\Sigma$; it carries $z=\beta$ into $\omega=i$ and $\Delta_{\rho}$ into a domain bounded by $\kappa_{\rho}$ and an $\operatorname{arc}$ of $|\omega|=1$ which contains $\omega=i$. Because of (1.1) this domain is contained in the disk $|\omega-i|<\lambda_{\rho}<\frac{\pi}{4}$ (since $\delta<e^{-32}$ ). Now the derivative of the inverse function of (1.2) is

$$
-\frac{d z}{d \omega}=\frac{2}{1-\omega^{2}}
$$

and for $|\omega-i|<\frac{\pi}{4}$

$$
\left|\frac{d z}{d \omega}\right| \leqq \frac{2}{\left(\sqrt{2}-\frac{\pi}{4}\right)^{2}}<\frac{2}{\left(\frac{6}{10}\right)^{2}}=\frac{50}{9}<6 .
$$

Consequently the length of $\gamma_{\rho}$ does not exceed $6 \lambda_{\rho}$ and hence the diameter of $\Delta_{\rho}$ is not larger than $6 \lambda_{\rho} \leqq M\left(\log \frac{1}{\delta}\right)^{-\frac{1}{2}}(M \leqq 6 \pi \sqrt{2})$. The proof is analogous when $\beta=b-i \frac{\pi}{2}$.

2. Semi-Conformality at the Boundary. We apply now Lemma 1 to give short proofs of two theorems on the distortion of the mapping $w \rightarrow Z(w)$ as $w \rightarrow w_{\infty}$. One of these is Ostrowski's [8, pp. 440-449] necessary and sufficient condition for "semi-conformality" at a boundary point, i.e. the existence of the $\lim _{w \rightarrow w_{\infty}}[Y(w)-v]$ in certain substrips of $S$, and the other concerns the oscillation of $X(w)$ on the segment $\theta_{u}$ as $u \rightarrow+\infty$.

We assume now that the domain $S$ defined in section 1 has the following additional properties:

A. For every $\Psi, 0<\Psi<\frac{\pi}{2}$, there exists an $R_{\Psi}$ such that the half-strip $S_{\Psi}:\left\{w=u+i v|| v \mid \leqq \Psi, u \geqq R_{\Psi}\right\}$ is contained in $S$.

B. There exist two sequences of boundary points $\left\{w_{n}=u_{n}+i v_{n}\right\}$ and 
$\left\{w_{n}^{*}=u_{n}^{*}+i v_{n}^{*}\right\}$ of $S$ such that

$$
\begin{aligned}
& u_{n+1}>u_{n}, u_{n} \rightarrow \infty, u_{n+1}-u_{n} \rightarrow 0 \text { and } v_{n} \rightarrow \frac{\pi}{2} \text { as } n \rightarrow \infty, \\
& u_{n+1}^{*}>u_{n}^{*}, u_{n}^{*} \rightarrow \infty, u_{n+1}^{*}-u_{n}^{*} \rightarrow 0 \text { and } v_{n}^{*} \rightarrow-\frac{\pi}{2} \text { as } n \rightarrow \infty .
\end{aligned}
$$

Remark. A domain $S$ which satisfies condition $A$ and, in addition, $B^{\prime}$ : $\lim _{u \rightarrow \infty} \theta(u)=\pi$ clearly satisfies condition $B$.

We prove first the sufficiency part of of Ostrowski's Theorem and some corollaries. The necessity part follows at the end of this section.

Theorem la (OSTROWSKI). Suppose $S$ is a domain with properties $A$ and B. If $Z(w)=X(w)+i Y(w)$ maps $S$ conformally onto the strip $\sum$ such that $\lim _{u \rightarrow+\infty} X(u)$ $=+\infty$, then uniformly in any half-strip $S_{\Psi}, 0<\Psi<\frac{\pi}{2}$,

$$
\lim _{u \rightarrow+\infty}(Y(w)-v)=0 \text {. }
$$

Corollaries. 1. Uniformly for $w \in S_{\Psi}, \lim _{u \rightarrow+\infty} Z^{\prime}(w)=1$.

2. Uniformly for $w_{1}=u+i v_{1} \in S_{\Psi}, w_{2}=u+i v_{2} \in S_{\Psi}$,

$$
\lim _{u \rightarrow+\infty}\left(X\left(w_{2}\right)-X\left(w_{1}\right)\right)=0 \text {. }
$$

3. For $t>u \geqq u_{0}:|X(t)-X(u)| \leqq(1+\varepsilon(u))(t-u)$ where $\lim _{u \rightarrow+\infty} \varepsilon(u)=0$.

Proof..$^{2)} \quad$ Let $\varepsilon, 0<\varepsilon<\frac{1}{3} e^{-64}$, be given, and suppose that

$$
S_{\frac{\pi}{2}-\varepsilon}:\left\{w=u+i v|| v \mid \leqq \frac{\pi}{2}-\varepsilon, u \geqq R(\varepsilon)\right\},
$$

where $R(\varepsilon)>u_{0}+1$, is contained in $S$. There exists an integer $N=N(\varepsilon)$ such that for $n \geqq N$

$$
u_{n+1}-u_{n}<\varepsilon, u_{n+1}^{*}-u_{n}^{*}<\varepsilon, v_{n}<\frac{\pi}{2}+\varepsilon, v_{n}^{*}>-\frac{\pi}{2}-\varepsilon .
$$

We assume that $u_{N}>R(\varepsilon), u_{N}^{*}>R(\varepsilon)$.

Let $\alpha_{n}$ be the endpoint of $\theta_{u_{n}}$ with $\operatorname{Im}\left(\alpha_{n}\right)>0$. Then clearly $\frac{\pi}{2}-\varepsilon<$ $\operatorname{Im}\left(\alpha_{n}\right)<\frac{\pi}{2}+\varepsilon$. The application of Lemma 1 to $\alpha_{n}$ with $^{3)} \delta^{2}=3 \varepsilon$ shows the

2) A proof of this theorem using Wolff's method was given by C. Gattegno [6]. Our proof, which is included here for the sake of completeness, seems somewhat simpler and allows us to obtain an additional Remark at the end of section 2 .

3) Note that the restriction on $\delta$ in Lemma 1 is satisfied uniformly for all $\alpha_{n}$ with $n \geqq N$. 
existence of a $\rho, \delta^{2}<\rho<\delta$, such that for $w \in D_{\rho}$

$$
\left|\frac{\pi}{2}-Y(w)\right| \leqq \frac{M}{\sqrt{\log \frac{1}{\delta}}}=\frac{M \sqrt{2}}{\sqrt{\log \frac{1}{3 \varepsilon}}}
$$

The circle $C_{\rho}$ about $\alpha_{n}$ intersects the line $v=\frac{\pi}{2}-\varepsilon$ (since the distance of $\alpha_{n}$ from this line is less than $2 \varepsilon$ and $\left.\rho \geqq \delta^{2}=3 \varepsilon\right)$. Hence $D_{\rho}$ contains a segment $s_{n}$ of this line, centered at $u_{n}+i\left(\begin{array}{c}\pi \\ 2\end{array}-\varepsilon\right)$ whose length is greater than $4 \varepsilon$. Because of $(2.1)$, the segments $s_{n}, s_{n+1}, \ldots \ldots(n \geqq N)$ overlap. Consequently (2.2) holds for all points $w$ on $v=\frac{\pi}{2}-\varepsilon$ for all $u \geqq u_{N}$. We have, therefore, for $v=\frac{\pi}{2}-\varepsilon$ and $u \geqq u_{N}$ :

$$
|Y(w)-v| \leqq \varepsilon+\frac{M \sqrt{2}}{\sqrt{\log \frac{1}{3 \varepsilon}}}
$$

An analogous argument shows that for $v=-\frac{\pi}{2}+\varepsilon, u \geqq u_{N}^{*}$ :

$$
|Y(w)-v| \leqq \varepsilon+\frac{M \sqrt{2}}{\sqrt{\log \frac{1}{3 \varepsilon}}} .
$$

Since $Y(w)-v$ is a bounded harmonic function in $S_{\frac{\pi}{2}-\varepsilon}$ it follows that uniformly in this half-strip

$$
\varlimsup_{u \rightarrow+\infty}|Y(w)-v| \leqq \varepsilon+\frac{M \sqrt{2}}{\sqrt{\log \frac{1}{3 \varepsilon}}}
$$

This inequality is a fortiori correct as $w \rightarrow \infty$ in any fixed half-strip $S_{\Psi}$, with $0<\Psi<\frac{\pi}{2}-\varepsilon$. We keep $\Psi$ fixed; since then $\varepsilon$ may be taken arbitrarily small it follows that

$$
\begin{aligned}
& \varlimsup_{\substack{u \rightarrow+\infty \\
|v| \leqq \Psi}}|Y(w)-v|=0 \\
& |v|
\end{aligned}
$$

and this proves the theorem.

Proof of Corollaries. Let $0<d<-\frac{1}{2}\left(\frac{\pi}{2}-\Psi\right)$. For $w \in S_{\Psi}$, 


$$
\left|Z^{\prime}(w)-1\right| \leqq \frac{2 h}{d} \text { where } h=\operatorname{Max}_{|t-w|=d}|Y(t)-\operatorname{Im}(t)| ;
$$

and $h \rightarrow 0$ as $w \rightarrow \infty$ in $S_{\Psi}$.

Corollaries 2 and 3 follow from 1 by use of the inequalities

$$
\begin{aligned}
\left|X\left(u+i v_{2}\right)-u-\left[X\left(u+i v_{1}\right)-u\right]\right| & =\left|\int_{v_{1}}^{v_{2}} \frac{\partial}{\partial v}[X(u+i v)-u] d v\right| \\
& \leqq \int_{v_{1}}^{v_{2}}\left|Z^{\prime}(u+i v)-1\right| d v,
\end{aligned}
$$

and

$$
|X(t)-X(u)| \leqq \int_{u}^{t}\left|Z^{\prime}(\xi)\right| d \xi
$$

Remark. If in Theorem $1 a$ the assumption $B$ is replaced by $B^{\prime}: \lim _{u \rightarrow \infty} \theta(u)=\pi$, then, for approach $w \rightarrow \infty$ in $K:\left\{w \mid w \in \theta_{u}, u>u_{0}\right\}$,

$$
\lim _{u \rightarrow \infty}(Y(w)-v)=0 \text {. }
$$

Proof. Let $\varepsilon, 0<\varepsilon<\frac{\pi}{8}$, be given and let $R=R(\varepsilon)>u_{0}$ be chosen such that

(a) $S_{\frac{\pi}{2}-\varepsilon}:\left\{w|| v \mid \leqq \frac{\pi}{2}-\varepsilon, u \geqq R\right\} \subset S$;

(b) for $w \in \theta_{u}, u>R:-\frac{\pi}{2}-\varepsilon<v<\frac{\pi}{2}+\varepsilon$ (using $A$ and $\left.B^{\prime}\right)$;

(c) $|Y(w)-v|<\varepsilon$ for $w \in S_{\frac{\pi}{2}-\varepsilon}$.

Thus $Z(w)$ maps $S_{\frac{\pi}{2}-\varepsilon}$ onto a subdomain of $\Sigma$ which contains the half-strip $\left\{z=x+i y|| y \mid \leqq \frac{\pi}{2}-2 \varepsilon, x \geqq \rho\right\}$. All points $w \in K$ with $|v|>\frac{\pi}{2}-\varepsilon$ and sufficiently large $u$, say $u \geqq R_{1} \geqq R$, are mapped, therefore, onto points $z=x+i y$ exterior to this strip and in $x \geqq \rho$. Consequently for such $w: \frac{\pi}{2}-2 \varepsilon \leqq|Y(w)|$ $<\frac{\pi}{2}$. Hence, for $w \in K$, with $\frac{\pi}{2}-\varepsilon<v<\frac{\pi}{2}+\varepsilon, u \geqq R_{1}$

$$
\varepsilon=\frac{\pi}{2}-\left(\frac{\pi}{2}-\varepsilon\right) \geqq Y(w)-v \geqq \frac{\pi}{2}-2 \varepsilon-\left(\frac{\pi}{2}+\varepsilon\right)=-3 \varepsilon
$$

and similarly, for $-\frac{\pi}{2}+\varepsilon>v>-\frac{\pi}{2}-\varepsilon, u \geqq R_{1}$

$$
-\varepsilon \leqq Y(w)-v \leqq 3 \varepsilon
$$

Thus by (c), (2.3) and (2.4), for all $w \in K, u \geqq R_{1}$ 


$$
|Y(w)-v|<3 \varepsilon
$$

We turn now to the necessity of Ostrowski's condition.

Theorem 1b. Suppose $w=W(z)=U(z)+i V(z), z=x+i y, \quad$ is a holomorphic univalent function in $\Sigma:\left\{|y|<\frac{\pi}{2}\right\}$ such that $U(x) \rightarrow+\infty$ as $x \rightarrow+\infty$ and that, uniformly in any substrip $|y| \leqq \Psi, 0<\Psi<\frac{\pi}{2}$ :

$$
V(z)-y \rightarrow 0, \text { as } x \rightarrow \infty \text {. }
$$

Then the image $S=W(\Sigma)$ possesses properties $A$ and $B$.

Proof. The condition (2.5) implies that $S$ possesses property $A$. Consequently $S$ has a boundary point $w_{\infty}$ at $w=\infty$ which is accessible along any curve in $|v| \leqq \Psi<\frac{\pi}{2}$. For the inverse function $z=Z(w)=X(w)+i Y(w)$ we then have $X(w) \rightarrow \infty$ and $Y(w)-v \rightarrow 0$ as $u \rightarrow \infty$, uniformly in any substrip $|v| \leqq \Psi<\frac{\pi}{2}$.

To show that $S$ has property $B$, suppose there exists a sequence $\left\{u_{n}\right\}, u_{n} \uparrow \infty$ as $n \rightarrow \infty$ such that for fixed $c>0$ and $\delta>0$, the rectangles $R_{n}:\left\{u_{n} \leqq u \leqq u_{n}+c\right.$, $\left.0 \leqq v \leqq \frac{\pi}{2}+\delta\right\}$ are contained in $S$. Let $\varepsilon>0, \varepsilon<-\frac{\pi}{4}$, be given and $N$ be so large that for $n>N$

$$
|Y(w)-v|<\varepsilon \text { for }|v| \leqq \frac{\pi}{2}-\varepsilon \text { and } u>u_{N} .
$$

Consider $v-Y(w)$ on $\partial R_{n}$. On the side

$$
s:\left\{u_{n} \leqq u \leqq u_{n}+c, v=\frac{\pi}{2}+\delta\right\}: v-Y(w) \geqq \frac{\pi}{2}+\delta-\frac{\pi}{2}=\delta .
$$

On the remainder of $\partial R_{n}$ we have $v-Y(w) \geqq-\varepsilon$, provided $u>u_{N}$. Map $R_{n}$ conformally onto $|\xi|<1$ such that $u_{n}+\frac{1}{2} c+i \frac{\pi}{4}$ correspond to $\xi=0$, and let $h(\xi)=v-Y(w), w \in R_{n} \cup \partial R_{n}$. Then

$$
h(0)=\frac{1}{2 \pi} \int_{0}^{2 \pi} h\left(e^{i t}\right) d t
$$

By (2.6) $h(0)<\varepsilon$ for $n>N$. If $\gamma$ is the length of the image of $s$ on $|\xi|=1$

$$
\varepsilon \geqq \frac{1}{2 \pi}(\delta \gamma-\varepsilon(2 \pi-\gamma)) \text {. }
$$


Since $\varepsilon$ may be arbitrarily small, we find $\delta \leqq 0$ contrary to our assumption.

Thus given any $\delta>0$ and any $\varepsilon>0$, for all sufficiently large $u$ every interval $[u, u+\varepsilon]$ must contain a point $u^{\prime}$ such that one endpoint of $\theta_{u},\left(u^{\prime}, v^{\prime}\right)$ satisfies the inequality $\frac{\pi}{2}-\varepsilon<v^{\prime}<\frac{\pi}{2}+\delta$, and, by an analogous argument, a point $\left(u^{*}, v^{*}\right)$ such that $-\frac{\pi}{2}+\varepsilon \geqq v^{*} \geqq-\frac{\pi}{2}-\delta$. Hence there exist sequences $\left(u_{n}, v_{n}\right)$ and $\left(u_{n}^{*}, v_{n}^{*}\right)$ satisfying condition $B$.

3. Distortion of the Mapping on $\theta_{u}$. We prove first a preliminary result.

Lemma 2. Suppose $S$ is a domain with properties $A$ and $B$. For $u>u_{0}, v>0$, let

$$
\tau_{+}(u)=\operatorname{Sup}_{u+i v \in \theta_{u}}|X(u+i v)-X(u)|, \tau_{-}(u)=\operatorname{Sup}_{u-i v \in \theta_{u}}|X(u-i v)-X(u)|
$$

Then

$$
\lim _{n \rightarrow \infty} \tau_{+}\left(u_{n}\right)=0, \lim _{n \rightarrow \infty} \tau_{-}\left(u_{n}^{*}\right)=0 \text {. }
$$

Proof. Let $\varepsilon, 0<\varepsilon<\frac{1}{3} e^{-64}$, be given, let $R>u_{0}$ be so chosen that (i) the strip

$$
S_{\frac{\pi}{2}-\varepsilon}=\left\{w=u+i v|| v \mid \leqq \frac{\pi}{2}-\varepsilon, \quad u>R\right\} \subset S .
$$

and (ii) $v_{n}<\frac{\pi}{2}+\varepsilon$ and $v_{n}^{*}>-\frac{\pi}{2}-\varepsilon$ for $u_{n}>R$ and $u_{n}^{*}>R$.

Let $\alpha_{n}$ be the endpoint of $\theta_{u_{n}}$ with $\operatorname{Im}\left(\alpha_{n}\right)>0$. Then $\frac{\pi}{2}-\varepsilon<\operatorname{Im}\left(\alpha_{n}\right) \leqq v_{n}$ $<\frac{\pi}{2}+\varepsilon$. For $u_{n}>R+1$, the application of Lemma 1 with $\delta^{2}=3 \varepsilon$ shows that for $w \in \theta_{u_{n}},\left|w-\alpha_{n}\right|<3 \varepsilon$

$$
\left|X(w)-X\left(\alpha_{n}^{\prime}\right)\right|<\frac{M \sqrt{2}}{\sqrt{\log \frac{1}{3 \varepsilon}}} \quad\left(\alpha_{n}^{\prime}=u_{n}+i\left(\frac{\pi}{2}-\varepsilon\right)\right) .
$$

By Corollary 2 of Theorem la, for any $w=u+i v$, with $|v| \leqq \frac{\pi}{2}-\varepsilon$

$$
|X(w)-X(u)|<\varepsilon,
$$

provided $u$ is sufficiently large. Combining these two inequalities we find for all sufficiently large $n, u_{n}+i v \in \theta_{u_{n}}, v \geqq 0$, 


$$
\left|X\left(u_{n}+i v\right)-X\left(u_{n}\right)\right|<\varepsilon+\frac{M \sqrt{2}}{\sqrt{\log -\frac{1}{3 \varepsilon}}} .
$$

Hence, for all sufficiently large $n$,

$$
\tau_{+}\left(u_{n}\right) \leqq \varepsilon+\frac{M / 2}{\sqrt{\log 3 \varepsilon}} .
$$

The proof of the statement on $\tau_{-}\left(u_{n}^{*}\right)$ is analogous.

Now we come to the principal theorem on the oscillation of $X(w)$ on $\theta_{u}$.

Theorem 2. Let $S$ be a domain with the properties $A$ and $B$. If for $u>u_{0}$

$$
\tau(u)=\operatorname{Sup}_{w_{k} \in \theta_{u}}\left|X\left(w_{2}\right)-X\left(w_{1}\right)\right| \quad(k=1,2)
$$

then

$$
\lim _{u \rightarrow \infty} \tau(u)=0 .
$$

Proof. Because of Corollary 2 of Theorem la it is sufficient to prove that

$$
\operatorname{Sup}_{\substack{w \in \theta_{u} \\|v| \geq \Psi}}|X(w)-X(u)| \rightarrow 0 \quad \text { as } u \rightarrow+\infty .
$$

We take in particular $\Psi=\frac{\pi}{4}$. Because of Theorem la there exists an $R \geqq u_{0}$ such that all points $w=u+i v$ on any $\theta_{u}$ with $u \geqq R$ and $|v| \geqq \frac{\pi}{4}$ are mapped by $Z(w)$ onto points $z=x+i y \in \Sigma$ with $|y| \geqq \frac{\pi}{8}$ and such that $v \geqq \frac{\pi}{4}$ entails $y \geqq \frac{\pi}{8}$ and $v \leqq-\frac{\pi}{4}$ implies $y \leqq-\frac{\pi}{8}$. Furthermore, the image of the part of the real axis with $u>R$ is contained in the strip $|y|<\frac{\pi}{8}$. Consequently, all points $z=x+i y$ in $\Sigma$ with $y>\frac{\pi}{8}\left(y<-\frac{\pi}{8}\right)$ and all sufficiently large $x$, say $x>\rho$, are carried by the inverse mapping into points of $S$ with $v>0$ $(v<0)$. We may assume that $R$ is so chosen that the images of all $\theta_{u}$ with $u>R$ lie in $x>\rho$.

Now let $\varepsilon, 0<\varepsilon<1$, be given and let for $n>N=N(\varepsilon)$

$$
u_{n+1}-u_{n}<\varepsilon, \quad u_{n+1}^{*}-u_{n}^{*}<\varepsilon
$$

and (by Lemma 2)

$$
\tau_{+}\left(u_{n}\right)<\varepsilon, \tau_{-}\left(u_{n}^{*}\right)<\varepsilon
$$


We may assume that $R$ is chosen so large that $u_{n}>R-1, u_{n}^{*}>R-1$ imply $n>N$. Then, for every $u>R$, the interval $[u, u+\varepsilon]$ contains a point $u_{n}$ and $u_{m}^{*}$ and the interval $[u-\varepsilon, u]$ a point $u_{k}$ and $u_{l}^{*}$, where $n, m, k, l>N$.

For every $w=u+i v$ with $u>R, v>\frac{\pi}{4}$ there exists a point $w_{n}^{\prime} \in \theta_{u_{n}}$ with $u \leqq u_{n} \leqq u+\varepsilon$ and $\operatorname{Im}\left(w_{n}^{\prime}\right)>0$ such that

$$
X(w) \leqq X\left(w_{n}^{\prime}\right)
$$

To find such a $w_{n}^{\prime}$ we consider on the image $\sigma_{u_{n}}$ of $\theta_{u_{n}}$ in the z-plane a point with the same ordinate as $Z(w)$. Such a point must have a larger abscissa than $X(w)$ and the ordinate $y=Y(w) \geqq \frac{\pi}{8}$. This point in turn will correspond in the w-plane to a $w_{n}^{\prime} \in \theta_{u_{n}}$ with $\operatorname{Im}\left(w_{n}^{\prime}\right)>0$. Now, by Lemma 2 ,

$$
X\left(w_{n}^{\prime}\right) \leqq X\left(u_{n}\right)+\tau_{+}\left(u_{n}\right) .
$$

By Corollary 3 of Theorem la, there exists a constant $c>1$ such that

$$
\left|X\left(u_{n}\right)-X(u)\right| \leqq c\left(u_{n}-u\right)
$$

and thus, combining (3.3), (3.4) and (3.5) we have

$$
X(w)-X(u)<c\left(u_{n}-u\right)+\tau_{+}\left(u_{n}\right) \leqq c \varepsilon+\varepsilon=(c+1) \varepsilon .
$$

In analogous way we obtain (choosing a point $u_{k}$ in $[u-\varepsilon, u]$ )

$$
X(w)-X(u)>-c \varepsilon-\tau_{+}\left(u_{k}\right)>-(c+1) \varepsilon .
$$

(Here use is made of the fact that $u_{k}>R-\varepsilon \geqq R-1$ and hence $k>N$ ). Thus

$$
\operatorname{Sup}_{\substack{w \in \theta_{u} \\ v \geq \frac{\pi}{4}}}|X(w)-X(u)| \leqq(c+1) \varepsilon .
$$

Similarly we show (using the points $u_{m}^{*}, u_{l}^{*}$ and $\tau_{-}(u)$ ) that

$$
\operatorname{Sup}_{\substack{w \in \theta_{u} \\ v \leqq-\frac{\pi}{4}}}|X(w)-X(u)| \leqq(c+1) \varepsilon .
$$

Hence (3.2) follows from (3.6) and (3.7).

4. Applications. Theorem 2 permits several applications. It yields a very short proof of Ostrowski's "Faltensatz" [8 p. 456], [7 p. 14] and leads to a new criterion for the differentiability of conformal maps at the boundary.

The "Faltensatz" is equivalent to the following theorem. 
Theorem 3. Suppose $S$ is a domain with the properties $A$ and $B$. If $w \in S$ let $u^{*}=$ inf $u$ for all $u \geqq u_{0}$ such that $\theta_{u}$ separates $w$ from $w_{\infty}$. Then

$$
\lim _{u^{*} \rightarrow+\infty}\left[X(w)-X\left(u^{*}\right)\right]=0^{4)} \text {. }
$$

Proof. If $w \in \theta u^{*}$, then the conclusion is that of Theorem 2. We may assume, therefore, that $w \notin \theta_{u^{*}}$. Then $\theta_{u^{*}}$ may or may not separate $w$ from $w_{\infty}$. We may assume that $u^{*}>u_{0}$.

If $\theta_{u^{*}}$ does separate $w$ from $w_{\infty}$ then

$$
X(w)<\operatorname{Max}_{t \in u_{u^{*}}} X(t) \leqq X\left(u^{*}\right)+\tau\left(u^{*}\right)
$$

by the definition of $\tau(u)$. Let $u<u^{*}$. Then, from the definition of $u^{*}, \theta_{u}$ does not separate $w$ from $w_{\infty}$. Hence

$$
X(w)>\min _{t \in \theta_{u}} X(t) \geqq X(u)-\tau(u) .
$$

Let $\delta>0$. Choose $u$ such that $0<u^{*}-u \leqq \delta$. Then

$$
X(u)-X\left(u^{*}\right)-\tau(u) \leqq X(w)-X\left(u^{*}\right) \leqq \tau\left(u^{*}\right) .
$$

By Corollary 3 of Theorem la there exists a constant $c>1$ such that

$$
\left|X(u)-X\left(u^{*}\right)\right| \leqq c\left|u-u^{*}\right| \leqq c \delta
$$

and thus

$$
-c \delta-\tau(u) \leqq X(w)-X\left(u^{*}\right) \leqq \tau\left(u^{*}\right) .
$$

The conclusion follows now from Theorem 2 and the fact that $\delta$ may be chosen arbitrarily small.

If $\theta_{u^{*}}$ does not separate $w$ from $w_{\infty}$, then

$$
X(w) \geqq \operatorname{Min}_{t \in u_{u^{*}}} X(t) \geqq X\left(u^{*}\right)-\tau\left(u^{*}\right)
$$

On the other hand, for $u>u^{*}, \theta_{u}$ must separate from $w_{\infty}$ and therefore

$$
X(w)<X(u)+\tau(u) .
$$

We complete now the proof in this case in an analogous manner.

We now turn to the second application.

Theorem 4. If $S$ is a strip with the properties $A$ and $B$, then for $w \in K$

4) The stipulation $u^{*} \rightarrow+\infty$ is equivalent to the statement: $w$ tends to the prime end containing $w_{\infty}$. 


$$
\lim _{u \rightarrow \infty}\left[X(w)-\pi \int_{u_{0}}^{u} \frac{d t}{\theta(t)}\right]=k
$$

exists and is either finite or $\left.+\infty .{ }^{5}\right)$

Proof. Suppose $w_{1}=u_{1}+i v_{1}, w_{2}=u_{2}+i v_{2}, u_{0}<u_{1}<u_{2}$, are in $K$. In the proof of Ahlfors' inequality [ 1 p. 8] it is first shown that

$$
\pi \int_{u_{1}}^{u_{2}} \frac{d u}{\theta(u)} \leqq \bar{x}_{2}-\underline{x}_{1}
$$

where

$$
\bar{x}_{2}=\sup _{w \in \theta_{u_{2}}} X(w), \quad \underline{x}_{1}=\inf _{w \in \theta_{u_{1}}} X(w) .
$$

Since $\bar{x}_{2} \leqq X\left(w_{2}\right)+\tau\left(u_{2}\right), \quad \underline{x}_{1} \geqq X\left(w_{1}\right)-\tau\left(u_{1}\right)$ we obtain

$$
\pi \int_{u_{1}}^{u_{2}} \frac{d u}{\theta(u)} \leqq X\left(w_{2}\right)-X\left(w_{1}\right)+\tau\left(u_{2}\right)+\tau\left(u_{1}\right)
$$

and $\tau(u) \rightarrow 0$ as $u \rightarrow \infty$, by Theorem 2. Thus, for $w=u+i v \in K, w_{1} \in K$,

$$
g(w) \equiv X(w)-\pi \int_{u_{0}}^{u} \frac{d t}{\theta(t)} \geqq X\left(w_{1}\right)-\pi \int_{u_{0}}^{u_{1}} \frac{d t}{\theta(t)}-\tau\left(u_{1}\right)-\tau(u) .
$$

Let $\bar{k}=\varlimsup_{u \rightarrow \infty} g(w), \quad \underline{k}=\overline{\lim }_{u \rightarrow \infty} g(w)$. Keeping $w_{1}$ fixed and letting $w \rightarrow w_{\infty}$ over a sequence in $K$ for which $g(w) \rightarrow \underline{k}$, we have

$$
\underline{k} \geqq g\left(w_{1}\right)-\tau\left(u_{1}\right)
$$

if we now let $w_{1} \rightarrow w_{\infty}$ such that $g\left(w_{1}\right) \rightarrow \bar{k}$, we obtain

$$
\underline{k} \geqq \bar{k} \text {. }
$$

Since also $\underline{k} \leqq \bar{k}$ we have $\bar{k}=\underline{k} ;(4.2)$ shows that $k>-\infty$, however, $\underline{k}$ and hence $k$ may be $+\infty$.

\section{Asymptotic Behavior of the Mapping Function in the Kernel.}

We now use Theorems 4 and la to obtain a result which yields a new criterion

5) After completion of this manuscript the author learned of a result of Dr. Barry G. Eke of the University of London. In his doctoral thesis, which is to be published shortly, Dr. Eke proves the existence of the limit (4.1) for more general domains than those satisfying the conditions for semiconformality at infinity. In view of the application we make below (Theorem $5)$ involving $\lim _{u \rightarrow \infty}(Z(w)-w)$, in which the semiconformality is essential, our proof of Theorem 4 seems of interest. 
for the differentiability of a conformal map at a boundary point.

Theorem 5. Suppose that $S$ is a domain with the property $A$ and that for $\varepsilon(u)=\operatorname{Max}(\theta(u)-\pi, 0)$,

$$
\left.\int_{u_{0}}^{\infty} \varepsilon(u) d u \text { converges }{ }^{6}\right) .
$$

Then for $w \in K$, the kernel of $S$,

$$
\lim _{u \rightarrow \infty}[X(w)-u]=\lambda
$$

exists and $\lambda$ is either finite or $+\infty$.

If $w \rightarrow w_{\infty}$ in any substrip $S_{\Psi}, 0<\Psi<\frac{\pi}{2}$, then

$$
\lim _{u \rightarrow \infty}[Z(w)-w]=\lambda
$$

Finally, if $\lim _{u \rightarrow \infty} \theta(u)=\pi$, then for $w \in K$

$$
\lim _{u \rightarrow \infty}[Z(w)-w]=\lambda
$$

Proof. We show first that $S$ satisfies property $B$. Let $\left\{\delta_{n}\right\}$ be a sequence such that $\delta_{n}>\delta_{n+1}, \quad \delta_{n} \rightarrow 0$ as $n \rightarrow \infty$, and $\Sigma / \sqrt{\delta_{n}}$ diverges. We determine a sequence $\left\{a_{n}\right\}, u_{0} \leqq a_{n}<a_{n+1}, a_{n} \rightarrow \infty$ as $n \rightarrow \infty$ such that $a_{n+1}-a_{n}>\sqrt{\delta_{n}}$ and

$$
\int_{a_{n}}^{\infty} \varepsilon(u) d u<\delta_{n}
$$

We partition $\left[a_{n}, a_{n+1}\right]$ by means of the points $a_{n}, a_{n}+\sqrt{\delta_{n}}, a_{n}+2 \sqrt{\delta_{n}}, \ldots \ldots$ $\ldots \ldots, a_{n}+m_{n} \sqrt{\delta_{n}}, a_{n+1}$, where $m_{n}$ is so chosen that

$$
\sqrt{\delta_{n}} \leqq a_{n+1}-\left(a_{n}+m_{n} \sqrt{\delta_{n}}\right)<2 \sqrt{\delta_{n}} .
$$

Denote the subintervals of $\left[a_{n}, a_{n+1}\right]$ by $i_{k, n}$; each has a length $\geqq \sqrt{\delta_{n}}$. We have,

$$
\int_{i_{k, n}} \varepsilon(u) d u \leqq \int_{a_{n}}^{\infty} \varepsilon(u) d u<\delta_{n},
$$

and there exists a $u_{k, n} \in i_{k, n}$ such that $\varepsilon\left(u_{k, n}\right)<\sqrt{\delta_{n}}$. We number the $\left\{u_{k, n}\right\}$ as a single sequence $u_{1}, u_{2}, u_{3}, \ldots \ldots$ which has the properties:

$$
u_{n+1}>u_{n}, u_{n} \rightarrow \infty, u_{n+1}-u_{n} \rightarrow 0 \text {, and } \varepsilon\left(u_{n}\right) \rightarrow 0 \text { as } n \rightarrow \infty \text {. }
$$

Let $v_{n}>0$ and $v_{n}^{\prime}<0$ denote the endpoints of $\theta_{u_{n}}$. Then

6) This does not imply that $\int_{u_{0}}^{\infty}(\theta(u)-\pi) d u$ converges. 


$$
\theta\left(u_{n}\right)=v_{n}+\left|v_{n}^{\prime}\right| \leqq \pi+\varepsilon\left(u_{n}\right)
$$

On the other hand, because of property $A, v_{n} \geqq \frac{\pi}{2}-\eta_{n},\left|v_{n}^{\prime}\right| \geqq \frac{\pi}{2}-\eta_{n}$ where $\eta_{\dot{n}} \rightarrow 0$ as $n \rightarrow \infty$. Hence

$$
\frac{\pi}{2}-\eta_{n} \leqq v_{n} \leqq \pi+\varepsilon\left(u_{n}\right)-\left|v_{n}^{\prime}\right| \leqq \frac{\pi}{2}+\varepsilon\left(u_{n}\right)+\eta_{n},
$$

and therefore $\lim _{n \rightarrow \infty} v_{n}=\frac{\pi}{2}$ and, similarly, $\lim _{n \rightarrow \infty} v_{n}^{\prime}=-\frac{\pi}{2}$. Thus $S$ has property $B$.

To complete the proof we write

$$
\begin{aligned}
X(w)-u & =X(w)-\pi \int_{u_{0}}^{u} \frac{d t}{\theta(t)}+\int_{u_{0}}^{u} \frac{\pi-\theta(t)}{\theta(t)} d t-u_{0} \\
& =g(w)-u_{0}+\int_{E_{+}} \frac{\pi-\theta}{\theta} d t+\int_{E_{-}} \frac{\pi-\theta}{\theta} d t
\end{aligned}
$$

where $E_{+}=\left\{t, u_{0} \leqq t \leqq u \mid \pi-\theta(t) \geqq 0\right\}, E_{-}=\left\{t, u_{0} \leqq t \leqq u \mid \pi-\theta(t)<0\right\}$. Since on $E_{-}: 0<\theta(t)-\pi=\varepsilon(t)$, it follows from (5.1) that

$$
\lim _{u \rightarrow \infty} \int_{E_{-}} \frac{\pi-\theta}{\theta} d t \text { exists and is finite. }
$$

As $u \rightarrow \infty, \int_{E_{+}} \frac{\pi-\theta}{\theta} d t$ either converges to a finite limit or to $+\infty$.

Hence

$$
\lim _{u \rightarrow \infty}[X(w)-u]=-u_{0}+\lim _{u \rightarrow \infty} g(w)+\lim _{u \rightarrow \infty} \int_{E_{+}} \frac{\pi-\theta}{\theta} d t+\lim _{u \rightarrow \infty} \int_{E_{-}} \frac{\pi-\theta}{\theta} d t=\lambda .
$$

The second part of the theorem, (5.3), follows from (5.2) and Theorem la, and the last part, (5.4), from (5.2) and the Remark to Theorem la at the end of Section 2.

Theorem 6. Suppose $S$ is a domain as in Theorem 5. Let for $u \geqq u_{0}, \beta(u)$ be a nonnegative continuous function such that

$$
\text { (i) } \int_{u_{0}}^{\infty} \beta(u) d u<\infty \text { and (ii) } \int_{u-\beta(u)}^{u+\beta(u)} \beta(t) d t \geqq c \beta^{2}(u)
$$

for a fixed $c>0$ and all sufficiently large $u{ }^{7)}$ If the strip $\left\{u \geqq u_{0},|v| \leqq \frac{\pi}{2}-\beta(u)\right\}$ is contained in $S$, then $\lambda<\infty$.

7) Condition (ii) is clearly satisfied if $\beta(u)$ is monotone for every $c \leqq 1$. Other examples for which (ii) holds are functions $\beta(u)$ such that for some constant $k>0$ either $\beta\left(u_{2}\right)-\beta\left(u_{1}\right) \geqq$ $-k\left(u_{2}-u_{1}\right)$ or $\beta\left(u_{2}\right)-\beta\left(u_{1}\right) \leqq k\left(u_{2}-u_{1}\right)$, where $u_{2}>u_{1}$. 
Proof. ${ }^{8)}$ First we map $S$ by means of $\omega=e^{-\left(w-i \frac{\pi}{2}\right)}$ onto a domain $\Omega$ in the $\omega$-plane, $\omega=\xi+i \eta$; then $w_{\infty}$ is transformed to the point $\omega_{0}$ at the origin, and $\omega_{0}$ is accessible in any angle $A: 0<\delta \leqq \arg \omega \leqq \pi-\delta, 0<\delta<\frac{\pi}{2}$. Let $(\varphi, \rho)$ denote polar coordinates in the $\omega$-plane, and let $\rho=e^{-u}, \beta_{1}(\rho)=\beta(-\log \rho)$ $=\beta(u) ; \beta_{1}(\rho)$ is continuous, and because of (ii) and (i) $\beta_{1}(\rho) \rightarrow 0$ as $\rho \rightarrow 0$; let $\beta_{1}(0)=0$. The arc $\gamma:\left\{\varphi=\beta_{1}(\rho), 0<\rho \leqq \rho_{0}=e^{-u_{\theta}}\right\}$ is contained in $\Omega$. Similarly, the arc $\gamma^{\prime}$ symmetrical to $\gamma$ with respect to the imaginary axis is in $\Omega$. We define $\beta_{1}(-t)=\beta_{1}(t), 0 \leqq t \leqq \rho_{0}$.

Let $C$ be a circle of radius $a>0$ about the point $a i$, where $2 a<\rho_{0}$. Let $\Gamma$ be arc of $C$ obtained by traversing $C$ from the point $2 a i$ in both directions to the first points of intersection with $\gamma$ and $\gamma^{\prime}$, respectively (at $\rho=\rho_{1}$, say). Name the subarcs of $\gamma$ and $\gamma^{\prime}$ from 0 to these points of intersection again $\gamma$ and $\gamma^{\prime}$. Then the domain $\Omega_{0}$ bounded by the closed Jordan curve $\gamma \cup \Gamma \cup \gamma^{\prime}$ contains $a i$ and is contained in $\Omega$. We may assume $\rho_{1}>0$; otherwise $\Gamma \equiv C$ and the conclusion of our theorem is well known. Also, we may assume $\beta_{1}(\rho)<\frac{\pi}{6}$ for $\rho \leqq \rho_{1}$.

Let $G_{0}(\omega, a i)=\log \frac{a}{r}-v(\omega)$ denote the Green's function for $\Omega_{0}$ with pole at $i a$, where $r=|\omega-i a|$ and $v(\omega)$ is harmonic in $\Omega_{0}$. Then for $\omega \in \partial \Omega_{0}$, we have $v(\omega)=\log \frac{a}{r}$.

Now, for $\omega \in \gamma, \omega=\rho e^{i \beta_{1}(\rho)}$

$$
\log \frac{a}{r}=\log \left(1+\frac{a-r}{r}\right)<\frac{a-r}{r} \leqq \frac{2}{a}(a-r) \leqq \frac{2}{a} \eta
$$

where $\eta=\operatorname{Im}(\omega)$. (Since $\beta_{1}(\rho)<\frac{\pi}{6}$ we have $r \geqq \frac{1}{2} a$ for $\omega \in \gamma$.)

Thus on $\gamma \cup \gamma^{\prime}$

$$
v(\omega) \leqq \frac{2}{a} \eta \quad(\eta=\operatorname{Im}(\omega))
$$

Consider now the harmonic function in $\operatorname{Im}(\omega)>0$

$$
h(\omega)=\frac{1}{\pi} \int_{-2 a}^{2 a}|t| \beta_{1}(t) \frac{\eta d t}{(\xi-t)^{2}+\eta^{2}} .
$$

We have for $\rho \leqq \rho_{1}$

8) The proof generalizes a method of M. Tsuji [9 p. 368]. 


$$
h(\omega) \geqq \frac{1}{\pi} \int_{\rho-2 \eta}^{\rho+2 \eta} t \beta_{1}(t) \frac{\eta}{(\xi-t)^{2}+\eta^{2}} d t \quad\left(\eta=\rho \sin \beta_{1}(\rho)\right) .
$$

Since in the interval of integration $|\xi-t|=\left|\rho \cos \beta_{1}(\rho)-t\right| \leqq 3 \eta$,

$$
h(\omega) \geqq \frac{1}{10 \pi \eta} \int_{\rho-2 \eta}^{\rho+2 \eta} t \beta_{1}(t) d t .
$$

If we set $\rho=e^{-u}$ and $t=e^{-\tau}$ a short calculation shows that the last integral is

$$
\geqq \int_{u-\beta(u)}^{u+\beta(u)} e^{-2 \tau} \beta(\tau) d \tau \geqq e^{-2 u} k \int_{u-\beta(u)}^{u+\beta(u)} \beta(\tau) d \tau \geqq k \cdot c \cdot \rho^{2} \beta_{1}{ }^{2}(\rho)
$$

by hypothesis (ii); $k$ is a positive constant. Hence for $\omega \in \gamma$

$$
h(\omega) \geqq \frac{b}{\eta} \rho^{2} \beta_{1}^{2}(\rho) \geqq b \eta
$$

where $b$ is a positive constant. The same estimate is obtained for $w \in \gamma^{\prime}$. Hence by (5.5) and (5.6) for $\omega \in \gamma \cup \gamma^{\prime}$

$$
v(\omega) \leqq \frac{2}{a} \eta \leqq \frac{2}{a b} h(\omega) .
$$

Furthermore $v(\omega)=0$ on $\Gamma$. Hence on $\partial \Omega_{0}$

$$
v(\omega) \leqq \frac{2}{a b} h(\omega)
$$

and therefore also in $\Omega_{0}$. Now, for $\omega=i \eta, 0<\eta<a$,

$$
h(i \eta)=\frac{1}{\pi} \int_{-2 a}^{2 a}|t| \beta_{1}(t) \frac{\eta}{t^{2}+\eta^{2}} d t \leqq \frac{\eta}{\pi} \int_{-2 a}^{2 a} \beta_{1}(t) \frac{d t}{|t|}
$$

and the last integral converges because of hypothesis (i). Thus by (5.7)

$$
v(i \eta) \leqq \frac{2}{a b} h(i \eta) \leqq \frac{2}{a b \pi} \eta \int_{-2 a}^{2 a} \beta_{1}(t) \frac{d t}{|t|}=A \eta .
$$

Next we have for $0<\eta<\frac{a}{2}$

$$
\begin{gathered}
G_{0}(i \eta, i a)=\log \frac{a}{r}-v(i \eta) \geqq \log \left(1+\frac{a-r}{r}\right)-A \eta \geqq \frac{1}{2} \frac{a-r}{a}-A \eta \\
\geqq \frac{\eta}{a}\left[\frac{1}{2}-\frac{2}{b \pi} \int_{-2 a}^{2 a} \beta_{1}(t) \frac{d t}{|t|}\right]=B \eta .
\end{gathered}
$$

We can choose a priori $a$ so small that $B>0$.

Let $G(\omega, i a)$ denote the Green's function of $\Omega$ with pole at $a i$. Since $\Omega_{0} \subset \Omega$ we have $G(\omega, i a) \geqq G_{0}(\omega, i a)$ and therefore 


$$
\lim _{\eta \rightarrow 0} \frac{G(i \eta, i a)}{\eta} \geqq \lim _{\eta \rightarrow 0} \frac{G_{0}(i \eta, i a)}{\eta} \geqq B>0
$$

If the map $Z(w)$ of $S$ onto $\Sigma$ is so normalized that the point in $S$ which corresponds to $i a \in \Omega$ is mapped onto $z=0$ then it is easily seen that $\lim _{\eta \rightarrow 0} \frac{G(i \eta, i a)}{\eta}=2 e^{-\lambda}$. Hence $\lambda \leqq \log _{B}{ }_{B}^{-}$.

Remark. Theorems 5 and 6 entail a criterion for the existence of the angular derivative of a conformal map at a boundary point. This criterion is stronger than those obtained earlier by Ahlfors [1, p. 36], the author [10], and by several others described in [7, pp. 22-28]. It is easily verified that our result implies these criteria. To show that it is actually stronger one might consider the domain $S$ which consists of the strip $|v|<\frac{\pi}{2}$ slit along the segments $\left\{u=u_{n},|v| \geqq \frac{\pi}{2}-\lambda_{n}\right\}$, with $u_{n} \uparrow \infty$ and $u_{n+1}-u_{n} \geqq \mathrm{c}>0[1, \mathrm{p} .39]$. The criteria cited do not permit one to decide whether the convergence of $\sum \lambda_{n}^{2}$ is sufficient for the existence of a finite limit (5.3). The question was answered in the affirmative by J. Wolff [12] with an $a d$ hoc proof, even without assuming the condition $u_{n+1}-u_{n} \geqq c$. A simple construction yields a function $\beta(u)$ as required in Theorem 6 and permits us to infer this result from Theorem 6 .

Our criterion also contains the more recent (and stronger) condition by J. Ferrand [3], Théorème p. 192. Other criteria by J. Dufresnoy and J. Ferrand [2, pp. 170-174] as well as related conditions in [5, pp. 208-211] apply to a wider class of domains but are weaker than ours in case the domain is contained in the strip $|v| \leqq \frac{\pi}{2}$.

\section{BIBLIOGRAPHY}

[1] L. Ahlfors, Untersuchungen zur Theorie der konformen Abbildung und der ganzen Funktionen, Acta Societatis Scientiarum Fennicae, nov. série A, vol. 1, No. 9 (1930), $1-40$.

[2] J. Dufresnoy et J. Ferrand, Extension d'une inégalité de M. Ahlfors et application au problème de la dérivée angulaire, Bull. des Sciences Math., 69 (1945), 165-174.

[ 3 ] J. Ferrand, Sur l'inégalité d'Ahlfors et son application au problème de la dérivée angulaire, Bulletin de la Societé Math de France, v. 72 (1944), 178-192.

[4] J. Lelong-Ferrand, Sur la représentation conforme des bandes, Journal d'Analyse Math. (Jerusalem) v. 2 (1952), 51-71.

[5] Représentation conforme et transformations a intégrale de Dirichlet bornée, Gauthier-Villars, Paris, 1955.

[6] C. Gattegno, Nouvelle démonstration d'un théorème de M. Ostrowski sur la représenta- 
tion conforme, Bull. des Sciences Math., 62 (1938), 12-21.

[7] C. Gattegno and A. Ostrowski, Représentation conforme à la frontière: domaines particuliers, Mémorial des Sciences Math., 110 (1949). Gauthier-Villars, Paris.

[ 8 ] A. Ostrowski, Zur Randverzerrung bei konformer Abbildung, Prace Mat. Fisycz. 44 (1936), 371-471.

[9] M. Tsuji, Potential Theory in Modern Function Theory, Maruzen Co., Ltd., Tokyo, 1959.

[10] S. Warschawski, Über das Randverhalten der Ableitung der Abbildungsfunktion bei konformer Abbildung, Math. Zeitschrift 35 (1932), 322-456.

[11] J. Wolff, Démonstration d'un théorème sur la conservation des angles dans la represéntation conforme au voisinage d'un point frontière, Proceedings, Kon. Akademie van Wetenschappen, Amsterdam, 38 (1935), 46-50.

[12] - Sur la représentation d'un demi-plan sur un demi-plan a une infinité d'incisions circulaires, Comptes Rendus, Acad. Sc. Paris, 200 (1935), 630-632.

University of California, San Diego, La Jolla, California, U.S.A. 